\title{
An asymptotically stable robust controller formulation for a class of MIMO nonlinear systems with uncertain dynamics
}

\author{
Baris Bidikli ${ }^{\mathrm{a}}$ Enver Tatlicioglu ${ }^{\mathrm{a}, *}$, Erkan Zergeroglu $^{\mathrm{b}}$ and Alper Bayrak ${ }^{\mathrm{c}}$ \\ ${ }^{a}$ Department of Electrical \& Electronics Engineering, Izmir Institute of Technology, Izmir, Turkey; ${ }^{b}$ Department of Computer \\ Engineering, Gebze Technical University, Gebze, Turkey; ${ }^{c}$ Department of Electrical \& Electronics Engineering, Abant Izzet Baysal \\ University, Bolu, Turkey
}

(Received 28 October 2014; accepted 7 April 2015)

\begin{abstract}
In this work, we present a novel continuous robust controller for a class of multi-input/multi-output nonlinear systems that contains unstructured uncertainties in their drift vectors and input matrices. The proposed controller compensates uncertainties in the system dynamics and achieves asymptotic tracking while requiring only the knowledge of the sign of the leading principal minors of the input gain matrix. A Lyapunov-based argument backed up with an integral inequality is applied to prove the asymptotic stability of the closed-loop system. Simulation results are presented to illustrate the viability of the proposed method.
\end{abstract}

Keywords: nonlinear uncertain dynamical systems; multi-input/multi-output systems; robust control; Lyapunov methods

\section{Nomenclature}

$$
\begin{array}{rl}
x(t) & \text { System states } \\
x^{(i)}(t) & i \text { th-order time derivative of } x(t) \\
H(\cdot), h(\cdot) & \text { Functional containing uncer- } \\
& \text { tain components } \\
G(\cdot), g(\cdot) & \text { Real-valued matrix with non- } \\
& \text { zero leading principal minors } \\
\tau(t), \tau_{1}(t), \tau_{2}(t) & \text { Control inputs } \\
X(t) & \text { Combined state vector } \\
S(X) & \text { Symmetric positive definite } \\
& \text { matrix } \\
D & \text { Diagonal matrix with entries } \\
& \pm 1 \\
U(X) & \text { Unity upper triangular matrix } \\
\dot{\tau}(t) & \text { Time derivative of control input } \\
\varphi\left(X, x^{(n)}\right) & \text { Auxiliary function } \\
m & \text { Positive bounding constant } \\
\bar{m}(X) & \text { Positive non-decreasing boun- } \\
& \text { ding function } \\
x_{r}(t) & \text { Reference trajectory } \\
e_{1}(t) & \text { Output tracking error } \\
e_{i}(t) & \text { Auxiliary error signals for } i= \\
& 2, \ldots, n \text { F } \\
r(t) & \text { Filtered error signal } \\
a_{i, j} & \text { Positive-valued coefficients } \\
& \text { generated via a Fibonacci num- } \\
& \text { ber series } \\
I_{m} & m \times m \text { identity matrix } \\
0_{m} \times 1 & \text { Vector of zeros } \\
&
\end{array}
$$

$$
\begin{aligned}
& N\left(X, x^{(n)}, t\right), \bar{N}(t), \tilde{N}(t) \quad \text { Auxiliary functions } \\
& X_{r}(t) \text { Combined vector of reference } \\
& \text { trajectory and its time deriva- } \\
& \text { tives } \\
& \Pi(t) \quad \text { Auxiliary signal } \\
& \alpha, K, C \text { Constant, diagonal, positive } \\
& \text { definite, gain matrices } \\
& \operatorname{Sgn}(\cdot) \quad \text { Vector signum function } \\
& \Lambda(t), \Phi(t), \Psi(t), \Theta(t) \quad \text { Auxiliary functions } \\
& \rho_{\widetilde{N}}(\cdot), \rho_{i, j}(\cdot) \quad \text { Non-negative, globally invert- } \\
& \text { ible, non-decreasing functions } \\
& z(t) \text { Combination of the error terms } \\
& \zeta_{\bar{N}_{i}}, \zeta_{\bar{U}_{i, j}}, \zeta_{\Theta}, \zeta_{\Omega_{i, j}} \quad \text { Positive bounding constants } \\
& \rho_{i}(\|z\|) \quad \text { Non-negative, globally invert- } \\
& \text { ible, non-decreasing functions } \\
& \Omega(\cdot) \quad \text { Strictly upper triangular matrix } \\
& \lambda_{\min }(\cdot) \text { Minimum eigenvalue of the } \\
& \text { gain matrix } \\
& \lambda_{1} \text { Positive bounding constant } \\
& \beta_{1} \quad \text { Positive constant } \\
& \gamma_{1}, \gamma_{2} \text { Positive bounding constants } \\
& \zeta_{L} \quad \text { Positive bounding constant } \\
& P(t) \quad \text { Auxiliary positive function } \\
& V(s, t) \quad \text { Auxiliary positive function } \\
& \beta \text { Positive constant }
\end{aligned}
$$

\section{Introduction}

This work focuses on the tracking control problem for multiinput/multi-output (MIMO) nonlinear uncertain systems.

\footnotetext{
${ }^{*}$ Corresponding author. Email: envertatlicioglu@iyte.edu.tr
} 
To our best knowledge, a generalised solution that covers all nonlinear systems for the aforementioned problem is not available. However, for special cases, there seems to be a great deal of results presented in the literature. To name a few, an adaptive backstepping method for strict feedback systems was utilised in Krstic, Kanellakopoulos, and Kokotovic (1995) with the assumption that the input gain matrix pre-multiplying the control input is known. In Kosmatopoulos and Ioannou (2002), a general procedure for the design of switching adaptive controllers including feedback linearisable and parametric-pure-feedback systems has been proposed. An adaptive neural controller for MIMO systems with block triangular form was proposed in Gee and Wang (2004).

Recently, in Wang, Chen, and Behal (2010), Wang, Behal, Xian, and Chen (2011), and Wang and Behal (2011), researchers have proposed robust- and adaptive-type controller formulations for the MIMO nonlinear systems of the following form:

$$
x^{(n)}=H\left(x, \dot{x}, \ldots, x^{(n-1)}\right)+G\left(x, \dot{x}, \ldots, x^{(n-2)}\right) \tau
$$

where $x^{(i)}(t) \in \mathbb{R}^{m \times 1} i=0, \ldots, n$, are the states with $(\cdot)^{(i)}$ denoting the $i$ th derivative with respect to time, $H(\cdot) \in \mathbb{R}^{m \times 1}$ and $G(\cdot) \in \mathbb{R}^{m \times m}$ are uncertain functions with $G(\cdot)$ being a real-valued matrix with non-zero leading principal minors, and $\tau(t) \in \mathbb{R}^{m \times 1}$ is the control input. Specifically, in Wang et al. (2010), authors have extended the work of Zhang, Dawson, de Queiroz, and Xian (2004) by redesigning the controller of Chen, Behal, and Dawson (2006) removing an algebraic loop and potential singularity in their previous design and obtained a global uniformly ultimately bounded (UUB) tracking error performance. In Wang et al. (2011), an adaptive controller that ensures asymptotic tracking has been proposed. Recently, a continuous robust controller achieving semi-global asymptotic tracking performance for uncertain MIMO systems of the form (1) with two degrees of freedom was proposed in Wang and Behal (2011).

In this work, we consider a broader class of uncertain MIMO nonlinear system than that of (1) which has the following form:

$$
x^{(n)}=h\left(x, \dot{x}, \ldots, x^{(n-1)}\right)+g\left(x, \dot{x}, \ldots, x^{(n-1)}\right) \tau
$$

where $x^{(i)}(t) \in \mathbb{R}^{m \times 1}, i=0, \ldots, n$, are the states, $h(\cdot) \in$ $\mathbb{R}^{m \times 1}$ is an uncertain function, $g(\cdot) \in \mathbb{R}^{m \times m}$ is an uncertain real-valued matrix with non-zero leading principal minors, and $\tau(t) \in \mathbb{R}^{m \times 1}$ is the control input. Example applications for the system model in (2) include, but not limited to, visual servoing (Zergeroglu, Dawson, de Queiroz, \& Behal, 2001), thermal management (Setlur, Wagner, Dawson, \& Chen, 2003), aeroelasticity vibration suppression (Reddy, Chen, Behal, \& Marzocca, 2007), and surface vessels (Lee, Tatlicioglu, Burg, \& Dawson, 2008).
When compared to (1), the dependence of $g(\cdot)$ on $x^{(n-1)}$ complicates the control design, and to our best knowledge, in the literature, there are only few works on this model. Namely, Xu and Ioannou (2003) considered the case where $g(\cdot)$ is either positive or negative definite, and designed a neural-network-based adaptive controller that ensured local convergence of the tracking error to a residual set. While Xian, Dawson, de Queiroz, and Chen (2004) considered the case where $g(\cdot)$ is positive definite, and a robust controller containing the integral of the signum of the error term was designed to obtain semi-global asymptotic tracking. More recently, Chen, Behal, and Dawson (2008) proposed a robust controller fused with a feedforward compensation term that ensured UUB tracking performance.

In this work, under similar restrictions given in Chen et al. (2008), we designed a new continuous robust controller for the class of nonlinear systems described by (2). Specifically, by applying a similar formulation to that of Xian et al. (2004), which is a nonlinear proportional integral controller fused with integral of the sign of the error feedback, we were able to achieve asymptotic tracking as opposed to the UUB tracking result of Chen et al. (2008). From this perspective, we might say that our work extends the results given in Xian et al. (2004) to a broader class of nonlinear systems. We would like to highlight that, due to the nature of the nonlinearities and uncertainties in the system given by (2), extending the results given in Xian et al. (2004) is not a straightforward task. Explicitly, Xian et al. (2004) considered the case where the input gain matrix $g(\cdot)$ being positive definite, while we consider the case where $g(\cdot)$ has non-zero leading principal minors. The results in Xian et al. (2004) can be considered as special cases of the results presented here. The stability analysis is conducted in four steps. First, we introduce an initial Lyapunov function to prove the boundedness of all the signals under the closed-loop system. Second, after utilising the boundedness of the error signals, an integral inequality is obtained. Third, a novel Lyapunov-like function is constructed and, via the use of the integral inequality, its nonnegativeness is proven. Finally, after fusing this Lyapunovlike function with the initial Lyapunov function (that was utilised to prove boundedness), asymptotic stability is proven.

The rest of the paper is organised as follows; Section 2 introduces the error system development while the controller development is presented in Section 3. Stability of the closed-loop system under the proposed method is investigated in Section 4, and the numerical simulations are given in Section 5. Finally, concluding remarks are presented in Section 6.

\section{Error system development}

The uncertain functions $h(\cdot)$ and $g(\cdot)$ of $(2)$ are assumed to be at least second-order differentiable (i.e., $h(\cdot), g(\cdot) \in \mathcal{C}^{n+2}$ ). 
Based on the assumption that $g(\cdot)$ being a real-valued matrix with non-zero leading principal minors, the following matrix decomposition is utilised (Costa, Hsu, Imai, \& Kokotovic, 2003; Morse, 1993):

$$
g=S(X) D U(X)
$$

where $X(t) \triangleq\left[\begin{array}{ll}x^{T} & \dot{x}^{T} \ldots\left(x^{(n-1)}\right)^{T}\end{array}\right]^{T} \in \mathbb{R}^{(m n) \times 1}$ is the combined state vector, $S(X) \in \mathbb{R}^{m \times m}$ is a symmetric positive definite matrix, $D \in \mathbb{R}^{m \times m}$ is a diagonal matrix with entries being \pm 1 , and $U(X) \in \mathbb{R}^{m \times m}$ is a unity upper triangular matrix. Similar to Chen et al. (2008) and Costa et al. (2003), we assume that $D$ is available for control design. We would like to note that since the leading principal minors of $g(X)$ are non-zero, $g^{-1}(X)$ exists and the following expression can be obtained by pre-multiplying (3) with $g^{-1}(X)$

$$
\tau=g^{-1}\left(x^{(n)}-h\right)
$$

Taking the time derivative of the system model in (2) and then substituting into (4) yields

$$
x^{(n+1)}=\varphi+S D U \dot{\tau}
$$

where (3) was utilised, and $\varphi\left(X, x^{(n)}\right) \in \mathbb{R}^{m \times 1}$ is an auxiliary function defined to have the following form:

$$
\varphi \triangleq \dot{h}+\dot{g} g^{-1}\left(x^{(n)}-h\right)
$$

Multiplying both sides of (5) with $S^{-1}(X)$ results in

$$
S^{-1} x^{(n+1)}=S^{-1} \varphi+D U \dot{\tau}
$$

and after defining $M(X) \triangleq S^{-1} \in \mathbb{R}^{m \times m}$ and $f\left(X, x^{(n)}\right) \triangleq$ $S^{-1} \varphi \in \mathbb{R}^{m \times 1}$, we obtain

$$
M x^{(n+1)}=f+D U \dot{\tau}
$$

It is noted that $M(X)$ satisfies the following inequalities:

$$
\underline{m}\|\chi\|^{2} \leq \chi^{T} M(X) \chi \leq \bar{m}(X)\|\chi\|^{2} \quad \forall \chi \in \mathbb{R}^{m \times 1}
$$

with $\underline{m} \in \mathbb{R}$ is a positive bounding constant, and $\bar{m}(X) \in \mathbb{R}$ is a positive, non-decreasing function.

Our control objective is to ensure that the system output $x(t)$ tracks a given smooth reference trajectory while ensuring all signals within the closed-loop system remain bounded. In order to quantify the tracking control objective, an error signal, $e_{1}(t) \in \mathbb{R}^{m \times 1}$, is defined to have the following form:

$$
e_{1} \triangleq x_{r}-x
$$

where $x_{r}(t) \in \mathbb{R}^{m \times 1}$ is the reference trajectory satisfying

$$
x_{r}(t) \in \mathcal{C}^{n+1}, x_{r}^{(i)}(t) \in \mathcal{L}_{\infty}, i=0,1, \ldots,(n+1)
$$

In our controller development, we will assume that the combined state vector $X(t)$ is available.

To facilitate the control design, auxiliary error signals, denoted by $e_{i}(t) \in \mathbb{R}^{m \times 1}, i=2, \ldots, n$, are defined as follows:

$$
\begin{aligned}
& e_{2} \triangleq \dot{e}_{1}+e_{1} \\
& e_{3} \triangleq \dot{e}_{2}+e_{2}+e_{1} \\
& \vdots \\
& e_{n} \triangleq \dot{e}_{n-1}+e_{n-1}+e_{n-2}
\end{aligned}
$$

A general expression for $e_{i}(t), i=2, \ldots, n$, in terms of $e_{1}(t)$ and its time derivatives can be obtained as follows:

$$
e_{i}=\sum_{j=0}^{i-1} a_{i, j} e_{1}^{(j)}
$$

where $a_{i, j} \in \mathbb{R}$ are known positive constants, generated via a Fibonacci number series (Xian et al., 2004). Our controller development also requires the definition of a filtered error signal, $r(t) \in \mathbb{R}^{m \times 1}$, which has the following form:

$$
r \triangleq \dot{e}_{n}+\alpha e_{n}
$$

where $\alpha \in \mathbb{R}^{m \times m}$ is a constant positive definite, diagonal, gain matrix. It should be noted that, since $\dot{e}_{n}(t)$ is unavailable, then $r(t)$ is also unavailable for control design. It should further be noted that the auxiliary error signals in (12)-(16) are introduced to obtain a stability analysis where only first-order time derivatives are utilised. After differentiating (16) and pre-multiplying the resulting equation with $M(X)$, the following expression can be derived:

$$
M \dot{r}=M\left(x_{r}^{(n+1)}+\sum_{j=0}^{n-2} a_{n, j} e_{1}^{(j+2)}+\alpha \dot{e}_{n}\right)-f-D U \dot{\tau}
$$

where (8), (10), (15), and the fact that $a_{n,(n-1)}=$ 1 were utilised. After defining an auxiliary function, $N\left(X, x^{(n)}, t\right) \in \mathbb{R}^{m \times 1}$,

$N \triangleq M\left(x_{r}^{(n+1)}+\sum_{j=0}^{n-2} a_{n, j} e_{1}^{(j+2)}+\alpha \dot{e}_{n}\right)-f+e_{n}+\frac{1}{2} \dot{M} r$ 
the expression in (17) can be reformulated to have the following form:

$$
M \dot{r}=-\frac{1}{2} \dot{M} r-e_{n}-D U \dot{\tau}+N
$$

Furthermore, the filtered error dynamics in (19) can be rearranged as

$$
M \dot{r}=-\frac{1}{2} \dot{M} r-e_{n}-D\left(U-I_{m}\right) \dot{\tau}-D \dot{\tau}+\widetilde{N}+\bar{N}
$$

where we added and subtracted $D \dot{\tau}(t)$ to the right-hand side, $I_{m} \in \mathbb{R}^{m \times m}$ is the standard identity matrix, and $\bar{N}(t)$, $\widetilde{N}(t) \in \mathbb{R}^{m \times 1}$ are auxiliary functions defined as follows: ${ }^{1}$

$$
\begin{gathered}
\left.\bar{N} \triangleq N\right|_{X=X_{r}, x^{(n)}=x_{r}^{(n)}} \\
\widetilde{N} \triangleq N-\bar{N}
\end{gathered}
$$

with $X_{r}(t) \triangleq\left[\begin{array}{lll}x_{r}^{T} & \dot{x}_{r}^{T} \ldots\left(x_{r}^{(n-1)}\right)^{T}\end{array}\right]^{T} \in \mathbb{R}^{(m n) \times 1}$ being a combination of the reference trajectory and its time derivatives.

The main idea behind adding and subtracting $D \dot{\tau}(t)$ term to the right-hand side of (20) is to make use of the fact that $U(X)$ is unity upper triangular, and thus $\left(U-I_{m}\right)$ is strictly upper triangular.

\section{Controller formulation}

Based on the open-loop error system in (20) and the subsequent stability analysis, the control input $\tau(t)$ is designed in the following form:

$$
\tau=D K\left[e_{n}(t)-e_{n}\left(t_{0}\right)+\alpha \int_{t_{0}}^{t} e_{n}(\sigma) d \sigma\right]+D \Pi
$$

where the auxiliary signal $\Pi(t) \in \mathbb{R}^{m \times 1}$ is generated according to the following equation:

$$
\dot{\Pi}=C \operatorname{Sgn}\left(e_{n}\right), \Pi\left(t_{0}\right)=0_{m \times 1}
$$

In (23) and (24), $K, C \in \mathbb{R}^{m \times m}$ are constant, diagonal, positive definite, gain matrices, $0_{m \times 1} \in \mathbb{R}^{m \times 1}$ is a vector of zeros and $\operatorname{Sgn}(\cdot) \in \mathbb{R}^{m \times 1}$ is the vector signum function. Notice that, for $i=n$ in (15), $e_{n}(t)$ and thus $\tau(t)$ depend on $x(t), \dot{x}(t), \ldots, x^{(n-1)}(t)$, and not $x^{(n)}(t)$. Based on the structures of (23) and (24), the following expression is obtained for the time derivative of the control input:

$$
\dot{\tau}=D K r+D C \operatorname{Sgn}\left(e_{n}\right)
$$

where (16) was utilised. The control gain is designed as $K=$ $I_{m}+k_{p} I_{m}+\operatorname{diag}\left\{k_{d, 1}, \ldots, k_{d,(m-1)}, 0\right\}$, where $k_{p}, k_{d, i} \in$ $\mathbb{R}$ are constant, positive, control gains, and $\operatorname{diag}\{\cdot\}$ is used to represent the entries of a diagonal matrix. Finally, after substituting (25) into (20), the closed-loop error system for $r(t)$ is obtained as

$$
\begin{aligned}
M \dot{r}= & -\frac{1}{2} \dot{M} r-e_{n}-K r+\widetilde{N}+\bar{N} \\
& -D\left(U-I_{m}\right) D K r-D U D C \operatorname{Sgn}\left(e_{n}\right)
\end{aligned}
$$

where the fact that $D D=I_{m}$ was utilised.

Before proceeding with the stability analysis, we would like to draw attention to the last two terms of (26) which we will investigate separately in the next two subsections.

\subsection{The $D\left(U-I_{m}\right) D K r$ term}

Note that, after utilising the fact that $\left(U-I_{m}\right)$ being strictly upper triangular, we rewrite the term $D\left(U-I_{m}\right) D K r$ as

$$
D\left(U-I_{m}\right) D K r=\left[\begin{array}{c}
\Lambda+\Phi \\
0
\end{array}\right]
$$

where $\Lambda(t), \Phi(t) \in \mathbb{R}^{(m-1) \times 1}$ are auxiliary functions with their entries $\Lambda_{i}(t), \Phi_{i}(t) \in \mathbb{R}, i=1, \ldots,(m-1)$, being defined as

$$
\begin{aligned}
& \Lambda_{i} \triangleq d_{i} \sum_{j=i+1}^{m} d_{j} k_{j} \widetilde{U}_{i, j} r_{j} \\
& \Phi_{i} \triangleq d_{i} \sum_{j=i+1}^{m} d_{j} k_{j} \bar{U}_{i, j} r_{j}
\end{aligned}
$$

with $\bar{U}_{i, j}\left(X_{r}\right), \widetilde{U}_{i, j}(t) \in \mathbb{R}$ are defined as

$$
\begin{gathered}
\left.\bar{U}_{i, j} \triangleq U_{i, j}\right|_{X=X_{r}} \\
\widetilde{U}_{i, j} \triangleq U_{i, j}-\bar{U}_{i, j}
\end{gathered}
$$

where $U_{i, j}(X) \in \mathbb{R}$ are the entries of $U(X)$. Notice from (27) that the last entry of the term $D\left(U-I_{m}\right) D K r$ is equal to 0 , and its $i$ th entry depends on the $(i+1)$ th to $m$ th entries of the control gain matrix $K$.

\subsection{The DUDCSgn $\left(e_{n}\right)$ term}

We rewrite the $D U D C \operatorname{Sgn}\left(e_{n}\right)$ term as

$$
D U D C \operatorname{Sgn}\left(e_{n}\right)=\left[\begin{array}{l}
\Psi \\
0
\end{array}\right]+\Theta
$$

where $\Psi(t) \in \mathbb{R}^{(m-1) \times 1}$ and $\Theta(t) \in \mathbb{R}^{m \times 1}$ are auxiliary functions defined as 


$$
\begin{gathered}
{\left[\begin{array}{l}
\Psi \\
0
\end{array}\right]=D(U-\bar{U}) D C \operatorname{Sgn}\left(e_{n}\right)} \\
\Theta=D \bar{U} D C \operatorname{Sgn}\left(e_{n}\right)
\end{gathered}
$$

where $\left.\bar{U}\left(X_{r}\right) \triangleq U\right|_{X=X_{r}} \in \mathbb{R}^{m \times m}$ is a function of reference trajectory and its time derivatives. The terms $\Psi_{i}(t) \in \mathbb{R}$, $i=1, \ldots,(m-1)$, and $\Theta_{i}(t) \in \mathbb{R}, i=1, \ldots, m$, are defined as

$$
\begin{gathered}
\Psi_{i} \triangleq d_{i} \sum_{j=i+1}^{m} d_{j} C_{j} \widetilde{U}_{i, j} \operatorname{sgn}\left(e_{n, j}\right) \\
\Theta_{i} \triangleq d_{i} \sum_{j=i}^{m} d_{j} C_{j} \bar{U}_{i, j} \operatorname{sgn}\left(e_{n, j}\right)
\end{gathered}
$$

Remark 1: The mean value theorem in Khalil (2002) can be utilised to develop the following upper bounds:

$$
\begin{gathered}
\|\widetilde{N}(t)\| \leq \rho_{\widetilde{N}}(\|z\|)\|z\| \\
\left\|\widetilde{U}_{i, j}(t)\right\| \leq \rho_{i, j}(\|z\|)\|z\|
\end{gathered}
$$

where $\rho_{\widetilde{N}}(\cdot), \rho_{i, j}(\cdot) \in \mathbb{R}$ are non-negative, globally invertible, non-decreasing functions of their arguments, and $z(t) \in \mathbb{R}^{[(n+1) m] \times 1}$ is defined by

$$
z \triangleq\left[\begin{array}{lllll}
e_{1}^{T} & e_{2}^{T} & \ldots & e_{n}^{T} & r^{T}
\end{array}\right]^{T}
$$

It can be seen from (11), (18), and (21) that $\bar{N}(t)$ and $\bar{U}_{i, j}(t)$ are bounded in the sense that

$$
\begin{aligned}
\left|\bar{N}_{i}(t)\right| & \leq \zeta_{\bar{N}_{i}} \\
\left|\bar{U}_{i, j}(t)\right| & \leq \zeta_{\bar{U}_{i, j}}
\end{aligned}
$$

$\forall t$ where $\zeta_{\bar{N}_{i}}, \zeta_{\bar{U}_{i, j}} \in \mathbb{R}$ are positive bounding constants. Based on (28), (29), (35), and (36), the following upper bounds can be obtained:

$$
\begin{aligned}
&\left|\Lambda_{i}\right| \leq \sum_{j=i+1}^{m} k_{j} \rho_{i, j}(\|z\|)\|z\|\left|r_{j}\right| \leq \rho_{\Lambda_{i}}(\|z\|)\|z\| \\
&\left|\Phi_{i}\right| \leq \sum_{j=i+1}^{m} k_{j} \zeta_{\bar{U}_{i, j}}\left|r_{j}\right| \leq \zeta_{\Phi_{i}}\|z\| \\
&\left|\Psi_{i}\right| \leq \sum_{j=i+1}^{m} C_{j} \rho_{i, j}(\|z\|)\|z\| \leq \rho_{\Psi_{i}}(\|z\|)\|z\| \\
&\left|\Theta_{i}\right| \leq \sum_{j=i}^{m} C_{j} \zeta_{\bar{U}_{i, j}} \leq \zeta_{\Theta_{i}}
\end{aligned}
$$

where (37)-(41) were utilised. From (45), it is easy to see that $\|\Theta(t)\| \leq \zeta_{\Theta} \forall t$ is satisfied for some positive bounding constant $\zeta_{\Theta} \in \mathbb{R}$, and from (42)-(44), we have

$$
\left|\Lambda_{i}\right|+\left|\Phi_{i}\right|+\left|\Psi_{i}\right| \leq \rho_{i}(\|z\|)\|z\|
$$

where $\rho_{i}(\|z\|) \in \mathbb{R} i=0,1, \ldots,(m-1)$, are non-negative, globally invertible, non-decreasing functions satisfying

$$
\rho_{\Lambda_{i}}+\rho_{\Psi_{i}}+\zeta_{\Phi_{i}} \leq \rho_{i}
$$

Remark 2: As a result of the fact that $\bar{U}(t)$ being unity upper triangular, $\Theta(t)$ in (34) can be rewritten as

$$
\Theta=\left(I_{m}+\Omega\right) C \operatorname{Sgn}\left(e_{n}\right)
$$

where $\Omega(t) \triangleq D\left(\bar{U}-I_{m}\right) D \in \mathbb{R}^{m \times m}$ is a strictly upper triangular matrix. Since it is a function of the reference trajectory and its time derivatives, its entries, denoted by $\Omega_{i, j}(t) \in \mathbb{R}$, are bounded in the sense that

$$
\left|\Omega_{i, j}(t)\right| \leq \zeta_{\Omega_{i, j}} \forall t
$$

where $\zeta_{\Omega_{i, j}} \in \mathbb{R}$ are positive bounding constants.

At this point, we are now ready to continue with the stability analysis of the proposed robust controller.

\section{Stability analysis}

In this section, via an initial Lyapunov-based analysis, we will first prove the boundedness of the error signals under the closed-loop operation. Using this result, we will then present a lemma and obtain an upper bound for the integral of the absolute values of the entries of $\dot{e}_{n}(t)$. This upper bound will later be utilised in another lemma to prove the non-negativity of a Lyapunov-like function that will be used in our final analysis which proves asymptotic stability of the tracking error.

Theorem 4.1: For the uncertain MIMO system of (2), the controller in (23) and (24) guarantees the boundedness of all the closed-loop signals including the error signals in (10), (12)-(14), and (16) provided that the control gains $k_{d, i}$ and $k_{p}$ are chosen large enough compared to the initial conditions of the system and the following condition is satisfied:

$$
\lambda_{\min }(\alpha) \geq \frac{1}{2}
$$

where the notation $\lambda_{\min }(\alpha)$ denotes the minimum eigenvalue of the gain matrix $\alpha$, previously introduced in (16). 
Proof: The non-negative function $V_{1}(z) \in \mathbb{R}$ is defined as

$$
V_{1} \triangleq \frac{1}{2} \sum_{i=1}^{n} e_{i}^{T} e_{i}+\frac{1}{2} r^{T} M r
$$

By utilising (9), (51) can be bounded in the following manner:2

$$
\frac{1}{2} \min \{1, \underline{m}\}\|z\|^{2} \leq V_{1}(z) \leq \frac{1}{2} \max \{1, \bar{m}(\|z\|)\}\|z\|^{2}
$$

where $z(t)$ was defined in (39), and the terms $\underline{m}, \bar{m}(\|z\|)$ were defined in (9). Taking the time derivative of (51) yields

$$
\dot{V}_{1}=\sum_{i=1}^{n} e_{i}^{T} \dot{e}_{i}+r^{T} M \dot{r}+\frac{1}{2} r^{T} \dot{M} r
$$

The first term in the above expression can be written as follows:

$$
\begin{aligned}
\sum_{i=1}^{n} e_{i}^{T} \dot{e}_{i}= & e_{1}^{T}\left(e_{2}-e_{1}\right)+e_{2}^{T}\left(e_{3}-e_{2}-e_{1}\right) \\
& +e_{3}^{T}\left(e_{4}-e_{3}-e_{2}\right)+\ldots \\
& +e_{n-1}^{T}\left(e_{n}-e_{n-1}-e_{n-2}\right)+e_{n}^{T}\left(r-\alpha e_{n}\right) \\
= & -\sum_{i=1}^{n-1} e_{i}^{T} e_{i}+e_{n-1}^{T} e_{n}+e_{n}^{T} r-e_{n}^{T} \alpha e_{n}
\end{aligned}
$$

where (12)-(14) and (16) were utilised. Substituting (26)(29), (32)-(34), and (54) into (53) results in

$$
\begin{aligned}
\dot{V}_{1}= & -\sum_{i=1}^{n-1} e_{i}^{T} e_{i}+e_{n-1}^{T} e_{n}+e_{n}^{T} r-e_{n}^{T} \alpha e_{n} \\
& +r^{T}\left(-\frac{1}{2} \dot{M} r-e_{n}-K r+\widetilde{N}+\bar{N}\right) \\
& -r^{T}\left[\begin{array}{c}
\Lambda+\Phi \\
0
\end{array}\right]-r^{T}\left[\begin{array}{c}
\Psi \\
0
\end{array}\right]-r^{T} \Theta+\frac{1}{2} r^{T} \dot{M} r
\end{aligned}
$$

which, after substituting the control gain matrix $K$, can be rewritten as

$$
\begin{aligned}
\dot{V}_{1}= & -\sum_{i=1}^{n-1} e_{i}^{T} e_{i}+e_{n-1}^{T} e_{n}-e_{n}^{T} \alpha e_{n}-r^{T} r \\
& +\left[r^{T} \tilde{N}-k_{p} r^{T} r\right] \\
& +\left[-\sum_{i=1}^{m-1} r_{i}\left(\Lambda_{i}+\Psi_{i}+\Phi_{i}\right)-\sum_{i=1}^{m-1} k_{d, i} r_{i}^{2}\right] \\
& +r^{T} \bar{N}-r^{T} \Theta .
\end{aligned}
$$

After completing the squares in bracketed terms, utilising $\|\bar{N}(t)\| \leq \zeta_{\bar{N}},\|\Theta(t)\| \leq \zeta_{\Theta}$ and $e_{n-1}^{T} e_{n} \leq 1 / 2\left\|e_{n-1}\right\|^{2}+$ $1 / 2\left\|e_{n}\right\|^{2}$, we obtain

$$
\begin{aligned}
\dot{V}_{1} \leq & -\sum_{i=1}^{n-2}\left\|e_{i}\right\|^{2}-\frac{1}{2}\left\|e_{n-1}\right\|^{2}-\left(\lambda \min (\alpha)-\frac{1}{2}\right)\left\|e_{n}\right\|^{2} \\
& -r^{T} r+\frac{\rho_{\widetilde{N}}^{2}(\|z\|)}{4 k_{p}}\|z\|^{2}+\sum_{i=1}^{m-1} \frac{\rho_{i}^{2}}{4 k_{d, i}}\|z\|^{2} \\
& +\|r\| \zeta_{\bar{N}}+\|r\| \zeta_{\Theta}
\end{aligned}
$$

which can then be rearranged as

$$
\dot{V}_{1} \leq-\left(\lambda_{1}-\frac{\rho_{\widetilde{N}}^{2}(\|z\|)}{4 k_{p}}-\sum_{i=1}^{m-1} \frac{\rho_{i}^{2}(\|z\|)}{4 k_{d, i}}\right)\|z\|^{2}+\delta \varepsilon^{2}
$$

where $\lambda_{1} \triangleq \min \left\{\frac{1}{2}, \lambda_{\min }(\alpha)-\frac{1}{2}, 1-\frac{1}{4 \delta}\right\}, \quad \delta \in \mathbb{R} \quad$ is a positive bounding constant, $\varepsilon \triangleq \zeta_{\bar{N}}+\zeta_{\Theta}$, and $\|r\| \varepsilon \leq$ $\frac{1}{4 \delta}\|r\|^{2}+\delta \varepsilon^{2}$ were utilised. Provided that the controller gains $k_{d, i}$ and $k_{p}$ are selected sufficiently large (larger than functions of the initial values of the norm of $z(t))$, we can ensure the terms presented in parenthesis in (58) are always positive, and utilising (52), the following inequality can be obtained:

$$
\dot{V}_{1} \leq-\beta_{1} V_{1}+\delta \varepsilon^{2}
$$

where $\beta_{1} \in \mathbb{R}$ is a positive constant. From (51) and (59), we can conclude that $V_{1}(t) \in \mathcal{L}_{\infty}$; therefore, $e_{i}(t), i=1, \ldots$, $n$, and $r(t)$ are UUB. Standard signal chasing arguments can then be utilised to prove that all the signals remain bounded under the closed-loop operation.

Remark 3: At this point, we would like to highlight the differences of the Lyapunov function used in the above proof and the one given in Equation (22) of Chen et al. (2008). While the structures of both are similar and the summation of the norm squares of the error signals $e_{i}(t), i=1, \ldots, n$, are the same, the second terms are fundamentally different (i.e., see Equation (16) in this paper and Equation (8) in Chen et al. (2008)).

Lemma 4.2: Provided that $e_{n}(t)$ and $\dot{e}_{n}(t)$ are bounded, the following expression for the upper bound of the integral of the absolute value of the ith entry of $\dot{e}_{n}(t), i=1, \ldots, m$, can be obtained:

$$
\int_{t_{0}}^{t}\left|\dot{e}_{n, i}(\sigma)\right| d \sigma \leq \gamma_{1}+\gamma_{2} \int_{t_{0}}^{t}\left|e_{n, i}(\sigma)\right| d \sigma+\left|e_{n, i}\right|
$$

where $\gamma_{1}, \gamma_{2} \in \mathbb{R}$ are some positive bounding constants.

Proof: While the proof is similar to that of the one given in Stepanyan and Kurdila (2009), it is presented in Appendix 1 for the sake of completeness. 
Lemma 4.3: Consider the term

$$
L \triangleq r^{T}\left(\bar{N}-\left(I_{m}+\Omega\right) C \operatorname{Sgn}\left(e_{n}\right)\right)
$$

where $\Omega(t)$ introduced in (48) is a strictly upper triangular matrix that is a function of reference trajectory and its time derivatives. Provided that the entries of the control gain $C$ are chosen to satisfy

$$
\begin{aligned}
C_{m} & \geq \zeta_{\bar{N}_{m}}\left(1+\frac{\gamma_{2}}{\alpha_{m}}\right) \\
C_{i} & \geq\left(\zeta_{\bar{N}_{i}}+\sum_{j=i+1}^{m} \zeta_{\Omega_{i, j}} C_{j}\right)\left(1+\frac{\gamma_{2}}{\alpha_{i}}\right), i=(m-1), \ldots, 1
\end{aligned}
$$

then it can be concluded that

$$
\int_{t_{0}}^{t} L(\sigma) d \sigma \leq \zeta_{L}
$$

where $\zeta_{L} \in \mathbb{R}$ is a positive bounding constant defined as

$$
\zeta_{L} \triangleq \gamma_{1} \sum_{i=1}^{m-1} \sum_{j=i+1}^{m} \zeta_{\Omega_{i, j}} C_{j}+\gamma_{1} \sum_{i=1}^{m} \zeta_{\bar{N}_{i}}+\sum_{i=1}^{m} C_{i}\left|e_{n, i}\left(t_{0}\right)\right|
$$

Proof: See Appendix 2.

Remark 4: At this point, we would like to highlight the differences of Lemma 4.3 in this paper and Lemma 1 in Xian et al. (2004). In Xian et al. (2004), the term $\operatorname{Sgn}\left(e_{n}\right)$ is multiplied with a constant gain matrix, while in (61), it is pre-multiplied with a time-varying uncertain matrix and then multiplied with a constant gain matrix. Thus, Lemma 1 in Xian et al. (2004) is a special case of Lemma 4.3 in this paper (i.e., when $\Omega(t)$ is a zero matrix). Additionally, the proof of Lemma 4.3 also removes the need of boundedness of $\|\dot{\bar{N}}(t)\|$, while Lemma 1 in Xian et al. (2004) requires it.

Theorem 4.4: Given the uncertain MIMO nonlinear system of the form (2), the controller of (23) and (24) ensures that the tracking error and its time derivatives converge to zero asymptotically in the sense that

$$
\left\|e_{1}^{(i)}(t)\right\| \rightarrow 0 \text { as } t \rightarrow+\infty, \forall i=0, \ldots, n
$$

provided that $\alpha$ is chosen to satisfy (50), the entries of $C$ are chosen to satisfy (62), and $k_{d, i}$ and $k_{p}$ are chosen large enough compared to the initial conditions of the system.

Proof: Let the auxiliary function $P(t) \in \mathbb{R}$ be defined as follows:

$$
P \triangleq \zeta_{L}-\int_{t_{0}}^{t} L(\sigma) d \sigma
$$

where the terms $\zeta_{L}$ and $L(t)$ were defined in (64) and (61), respectively, and when the entries of the control gain matrix $C$ are chosen to satisfy (62), from the proof of Lemma 4.3, we can conclude that $P(t)$ is non-negative. At this stage, consider the Lyapunov function, denoted by $V(s, t) \in \mathbb{R}$, defined as follows:

$$
V \triangleq V_{1}+P
$$

where $s(t) \in \mathbb{R}^{[(n+1) m+1] \times 1}$ is defined as

$$
s \triangleq\left[z^{T} \sqrt{P}\right]^{T}
$$

and $V_{1}(t) \in \mathbb{R}$ was defined in the proof of Theorem 4.1. By utilising (9), (66) can be upper and lower bounded in the following form:

$$
W_{1}(s) \leq V(s, t) \leq W_{2}(s)
$$

where $W_{1}(s), W_{2}(s) \in \mathbb{R}$ are defined as

$$
W_{1} \triangleq \lambda_{2}\|s\|^{2}, W_{2} \triangleq \lambda_{3}(\|s\|)\|s\|^{2}
$$

with $\lambda_{2} \triangleq \frac{1}{2} \min \{1, \underline{m}\}$ and $\lambda_{3} \triangleq \max \left\{1, \frac{1}{2} \bar{m}(\|z\|)\right\}$.

Taking the time derivative of $V(t)$, utilising the time derivative of (63), cancelling the common terms and following similar steps to that of proof of Theorem 4.1 yields

$$
\begin{aligned}
\dot{V}= & -\sum_{i=1}^{n-1} e_{i}^{T} e_{i}+e_{n-1}^{T} e_{n}-e_{n}^{T} \alpha e_{n}-r^{T} r+\left[r^{T} \tilde{N}-k_{p} r^{T} r\right] \\
& +\left[-\sum_{i=1}^{m-1} r_{i}\left(\Lambda_{i}+\Psi_{i}+\Phi_{i}\right)-\sum_{i=1}^{m-1} k_{d, i} r_{i}^{2}\right]
\end{aligned}
$$

which can be rearranged to have the following form:

$$
\begin{aligned}
\dot{V} \leq & -\sum_{i=1}^{n-2}\left\|e_{i}\right\|^{2}-\frac{1}{2}\left\|e_{n-1}\right\|^{2}-\left(\lambda_{\min }(\alpha)-\frac{1}{2}\right)\left\|e_{n}\right\|^{2} \\
& -r^{T} r+\frac{\rho_{\widetilde{N}}^{2}(\|z\|)}{4 k_{p}}\|z\|^{2}+\sum_{i=1}^{m-1} \frac{\rho_{i}^{2}(\|z\|)}{4 k_{d, i}}\|z\|^{2} \\
& \leq-\left(\lambda_{4}-\frac{\rho_{\widetilde{N}}^{2}(\|z\|)}{4 k_{p}}-\sum_{i=1}^{m-1} \frac{\rho_{i}^{2}(\|z\|)}{4 k_{d, i}}\right)\|z\|^{2}
\end{aligned}
$$

where $\lambda_{4} \triangleq \min \left\{\frac{1}{2}, \lambda_{\min }(\alpha)-\frac{1}{2}\right\}$. When the controller gains $k_{p}$ and $k_{d, i}$ for $i=1, \ldots,(m-1)$ are selected large enough such that the regions defined by $\mathcal{D}_{z} \triangleq$ $\{z:\|z\| \leq \mathcal{R}\}$ and $\mathcal{D}_{s} \triangleq\{s:\|s\| \leq \mathcal{R}\}$ with $\mathcal{R}$ defined as

$$
\mathcal{R}=\min \left\{\rho_{\widetilde{N}}^{-1}\left(2 \sqrt{k_{p} \frac{1-\beta}{m}}\right), \rho_{i}^{-1}\left(2 \sqrt{k_{d, i} \frac{1-\beta}{m}}\right)\right\}
$$



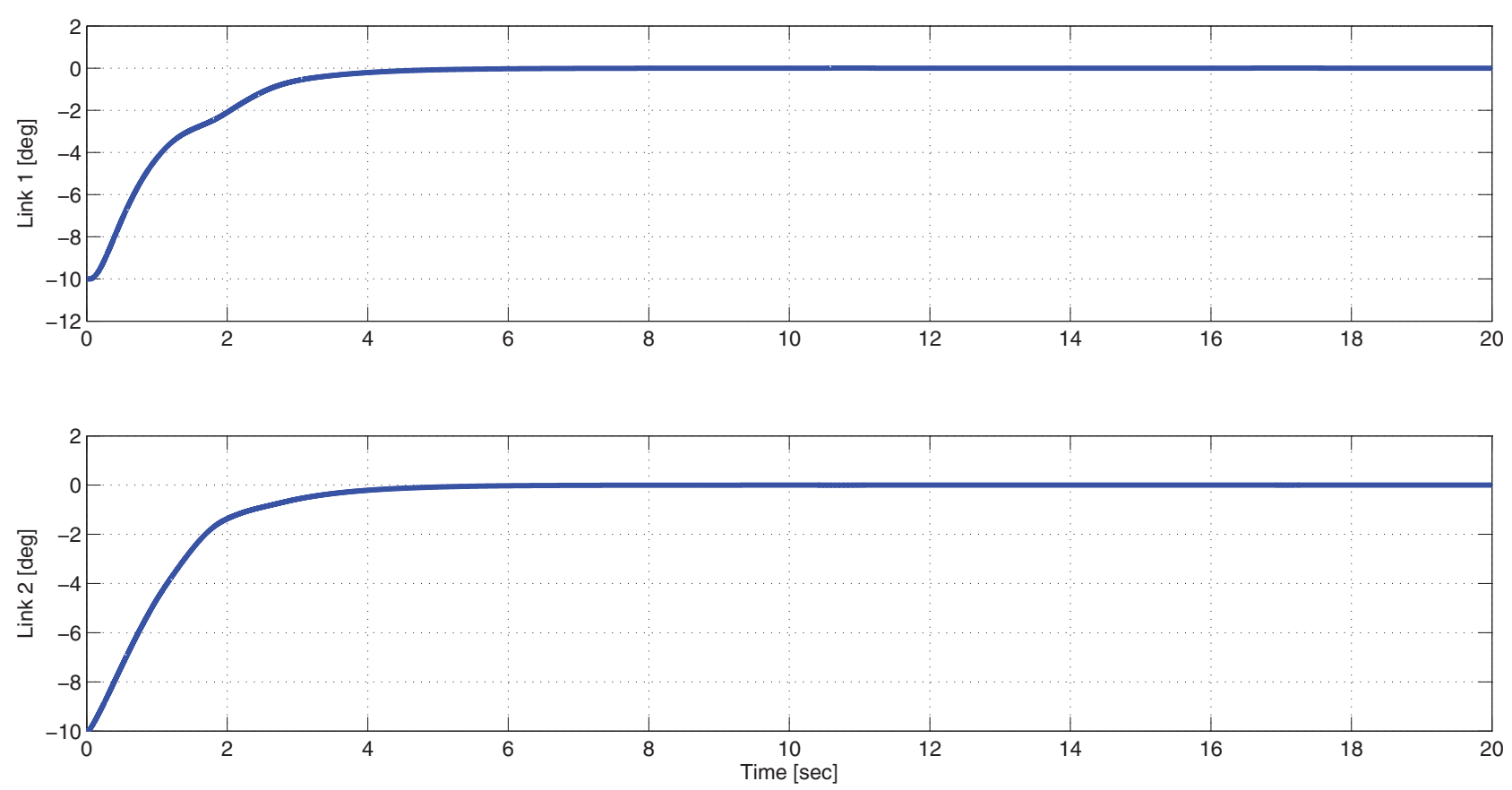

Figure 1. Link tracking errors.

for $i=1, \ldots,(m-1)$ are non-empty, from (72) and the definition of $s$, one can restate

$$
\dot{V} \leq-\beta\|z\|^{2}=-W(s), \forall s \in \mathcal{D}_{s}
$$

where $\beta \in \mathbb{R}$ is a positive constant that satisfies $0 \leq \beta$ $<1$. From (66) and (74), it is obvious that $V(t) \in \mathcal{L}_{\infty}$, and from the proof of Theorem 4.1, we concluded that all signals in the closed-loop error system are bounded, and furthermore, from the boundedness of $\dot{W}(s)$, we can state $W(s)$ is uniformly continuous.

Based on the definition of $\mathcal{D}_{s}$, another region, $\mathcal{S}$, can be defined in the following form:

$$
\begin{aligned}
& \mathcal{S} \triangleq\left\{s \in \mathcal{D}_{s}: W_{2}(s)<\lambda_{3}\left(\rho_{\tilde{N}}^{-1}\left(2 \sqrt{k_{p} \frac{1-\beta}{m}}\right)\right)^{2}\right\} \\
& \cap\left\{s \in \mathcal{D}_{s}: W_{2}(s)<\lambda_{3}\left(\rho_{1}^{-1}\left(2 \sqrt{k_{d, 1} \frac{1-\beta}{m}}\right)\right)^{2}\right\} \\
& \cap \vdots \\
& \cap\left\{s \in \mathcal{D}_{s}: W_{2}(s)<\lambda_{3}\left(\rho_{(m-1)}^{-1}\left(2 \sqrt{k_{d,(m-1)} \frac{1-\beta}{m}}\right)\right)^{2}\right\}
\end{aligned}
$$

A direct application of Theorem 8.4 in (Khalil, 2002) can be used to prove that $\|z(t)\| \rightarrow 0$ as $t \rightarrow+\infty \forall s\left(t_{0}\right) \in \mathcal{S}$. Based on the definition of $z(t)$, it is easy to show that $\left\|e_{i}(t)\right\|$, $\|r(t)\| \rightarrow 0$ as $t \rightarrow+\infty \forall s\left(t_{0}\right) \in \mathcal{S}, i=1, \ldots, n$. From (16), it is clear that $\left\|\dot{e}_{n}(t)\right\| \rightarrow 0$ as $t \rightarrow+\infty \forall s\left(t_{0}\right) \in \mathcal{S}$. By utilising (15) recursively, it can be proven that $\left\|e_{1}^{(i)}(t)\right\| \rightarrow$ 0 as $t \rightarrow+\infty, i=1, \ldots, n \forall s\left(t_{0}\right) \in \mathcal{S}$. Note that the region of attraction can be made arbitrarily large to include any initial conditions by choosing the controller gains $k_{p}$ and $k_{d, i}, i=1, \ldots,(m-1)$. This fact implies that the stability result obtained by the proposed method is semi-global.

Remark 5: The entries of the control gain matrix $C$ are required to satisfy (62) which depends on the constant upper bounds of uncertain system functions, and the entries of the control gain matrix $K$ are required to be chosen large enough compared to the initial conditions of the system. While this is a weakness of the controller, we will address this issue by utilising the self-tuning strategy that we recently designed in Bidikli, Tatlicioglu, Bayrak, and Zergeroglu (2013) and Bidikli, Tatlicioglu, and Zergeroglu (2014) for the family of the controllers in Xian et al. (2004) as an add-on to adjust the entries of $C$ and $K$.

\section{Numerical results}

To backup the theoretical results presented in the previous sections, some comparative numerical studies have been carried out. Similar to Chen et al. (2008), the performance and liability of the proposed nonlinear robust controller have been tested on a modified version of the two-link robot manipulator system with coupling between the two links taken from Slotine and Li (1991). The equations of motion are given as (Chen et al., 2008)

$$
\begin{aligned}
& {\left[\begin{array}{l}
\ddot{q}_{1} \\
\ddot{q}_{2}
\end{array}\right]=\left[\begin{array}{ll}
M_{11} & M_{12} \\
M_{12} & M_{22}
\end{array}\right]^{-1}\left[\begin{array}{l}
-b \dot{q}_{2}-b\left(\dot{q}_{1}+\dot{q}_{2}\right) \\
-b \dot{q}_{1} 0
\end{array}\right]\left[\begin{array}{l}
\dot{q}_{1} \\
\dot{q}_{2}
\end{array}\right]} \\
& +\left[\begin{array}{ll}
M_{11} & M_{12} \\
M_{12} & M_{22}
\end{array}\right]^{-1}\left[\begin{array}{ll}
1 & 1 \\
0 & 1
\end{array}\right]\left[\begin{array}{l}
\tau_{1} \\
\tau_{2}
\end{array}\right]
\end{aligned}
$$



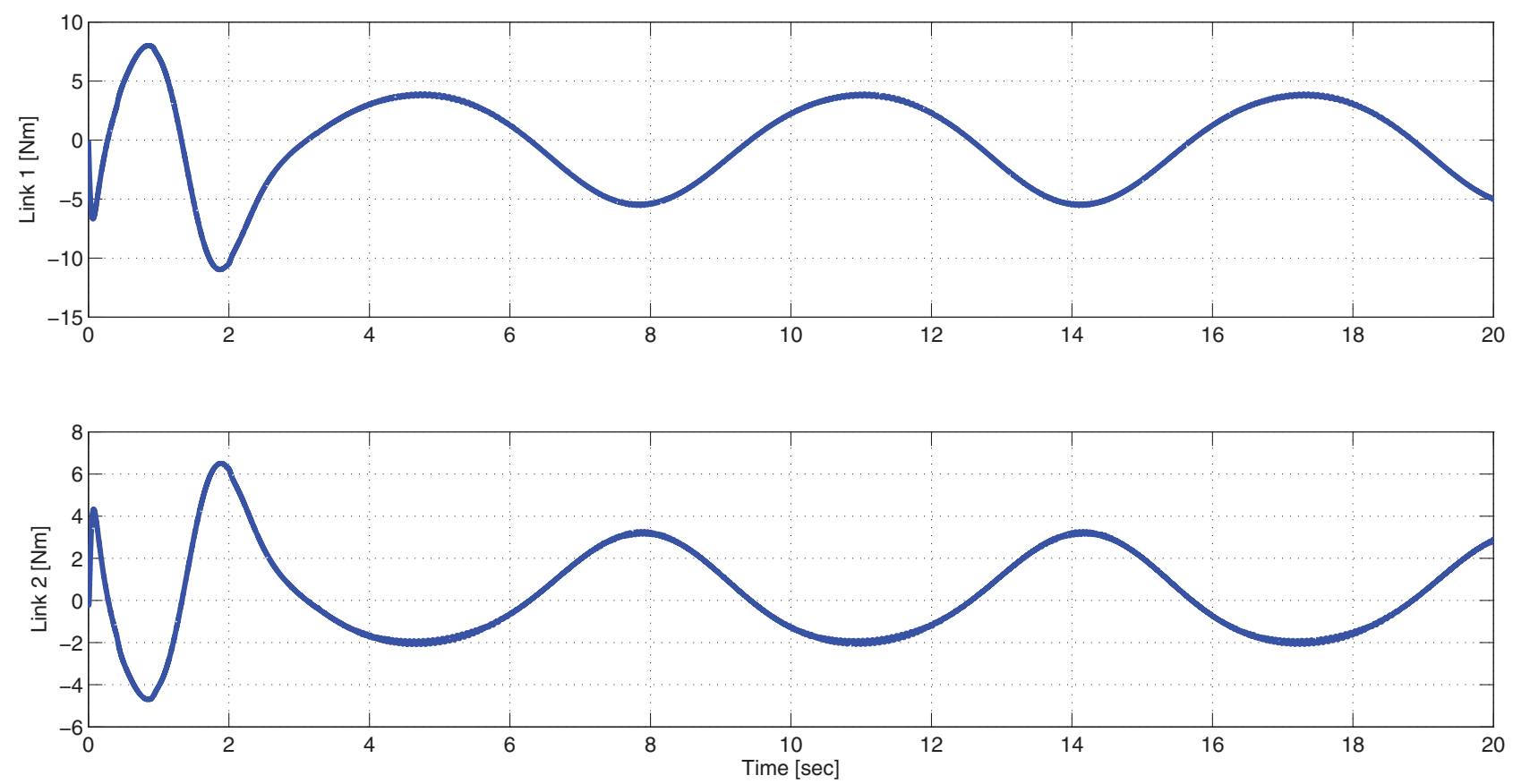

Figure 2. Control inputs.

where $q_{1}(t), q_{2}(t) \in \mathbb{R}$ denote the joint angles, $\tau_{1}(t)$ and $\tau_{2}(t)$ are the control inputs, and $M_{11}, M_{12}, M_{22}$, and $b$ are explicitly defined as

$$
\begin{aligned}
& M_{11}=a_{1}+2 a_{3} \cos q_{2}+2 a_{4} \sin q_{2}+a_{5}\left(\cos \dot{q}_{2}+\sin \dot{q}_{2}\right) \\
& +a_{6}\left(\cos q_{1}+\sin q_{1}\right) \\
& M_{12}=a_{2}+a_{3} \cos q_{2}+a_{4} \sin q_{2} \\
& M_{22}=a_{2}+a_{7}\left(\cos \dot{q}_{1}+\sin \dot{q}_{1}\right) \\
& b=a_{3} \sin q_{2}-a_{4} \sin q_{2}
\end{aligned}
$$

where $a_{1}=4.42, a_{2}=0.97, a_{3}=1.04, a_{4}=0.6, a_{5}=0.25$, $a_{6}=0.2$, and $a_{7}=0.5$. It can be seen from (76) and (77)(80) that the given model reflects the behaviour of the model in (1) as it is in the form of (2) with the decomposition in (3). The control objective is to make $q_{1}(t)$ and $q_{2}(t)$ follow a sinusoidal reference trajectory chosen as

$$
q_{d}(t)=\left(1-\exp \left(-0.3 t^{3}\right)\right)[30 \sin (t) 45 \sin (t)]^{T}(\mathrm{deg})
$$

As mentioned in Remark 3, the entries of the control gain matrices $C$ and $K$ are adjusted by the self-tuning method in Bidikli et al. (2013) and Bidikli et al. (2014), and the gains obtained were

$$
\alpha=\operatorname{diag}\{1,5\}, K=\operatorname{diag}\{175,125\}, C=\operatorname{diag}\{5,5\}
$$

The link position tracking error is depicted in Figure 1, while the control input is shown in Figure 2. From Figure 1, it is clear that the tracking control objective was successfully met.

\section{Conclusion}

In this work, we presented a continuous nonlinear robust controller designed for a class of uncertain MIMO nonlinear systems having non-zero leading principal minors in their input gain matrices. The stability of the closed-loop system was investigated via the use of Lyapunov-based arguments. Specifically, we developed a four-step analysis to prove the asymptotic stability of the output tracking error and its time derivatives. The results are also demonstrated via numerical simulations to illustrate the viability and performance of the proposed controller.

We would like to compare our result with some of the closest robust control works in the literature. After considering the same class of uncertain MIMO nonlinear system and imposing the same assumptions on the system model, we extended the results in Chen et al. (2008) to asymptotic as opposed to their UUB result. Similarly, in Xian et al. (2004), the same system model was considered. We extended the results in Xian et al. (2004) by relaxing the positive definiteness of the input gain matrix. After this, the results in (Xian et al., 2004) can now be considered 
as a special case of our controller (i.e., when $D U(X)$ is an identity matrix).

While a robust control strategy is employed in this work to compensate for uncertainties, adaptive methods can also be utilised. It is apparent that robust controllers rely on worst case scenario and thus require more control efforts. On the other hand, adaptive (or neural-network-based) methods can reduce the control effort by learning some, if not all, of the model uncertainties.

Our current research studies are devoted to designing an adaptive extension of the proposed work. It is noted that due to both matrix decomposition and matrix inverse operation, the adaptive version of this study is non-trivial.

We would like to compare our work with Xian et al. (2004) by performing experiments. Experimental verification on a robot manipulator can be performed by multiplying the control input torque with a matrix similar to that of the one in (76).

\section{Disclosure statement}

No potential conflict of interest was reported by the authors.

\section{Funding}

Work of E. Tatlicioglu is supported in part by The Scientific and Technological Research Council of Turkey [grant number 113E147].

\section{Notes}

1. The notation (.) is preferred to denote a function that depends on the reference trajectory and its time derivatives, and $\widetilde{(\cdot)}$ denotes a function that can be upper bounded by functions of the error signals.

2. Using (10) and (12)-(14), it can be shown that $\left\|\left(x, \dot{x}, \ldots, x^{n-1}\right)\right\| \leq \psi(\|z\|)$ where $\psi(\cdot)$ is some positive function. Thus, $\bar{m}\left(x, \dot{x}, \ldots, x^{n-1}\right) \leq \bar{m}(\|z\|)$.

\section{Notes on contributors}

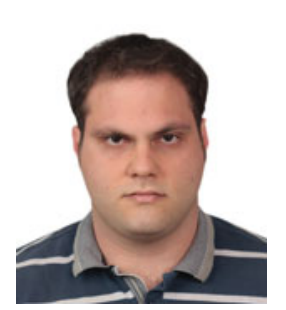

Baris Bidikli received his BSc degree in Electrical \& Electronics Engineering from Pamukkale University, Denizli, Turkey, and his MSc degree in Electrical \& Electronics Engineering from Izmir Institute of Technology, Izmir, Turkey, in 2011 and 2013, respectively. Since 2013, he has continued his $\mathrm{PhD}$ degree at Department of Electrical \& Electronics Engineering at Izmir Institute of Technology, Izmir, Turkey, where he is currently a research assistant. His research interests include estimation techniques, observer design, adaptive, robust, and optimal control of uncertain nonlinear systems, partial state feedback and output feedback control techniques, nonlinear control techniques for mechatronic systems, dynamic modelling and dynamic simulation of mechatronic systems, and swarm robotic applications.

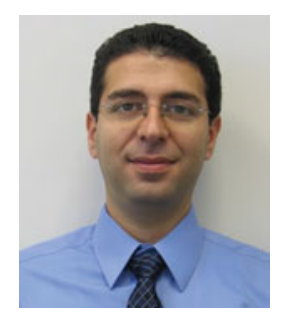

Enver Tatlicioglu received his $\mathrm{BSc}$ degree in Electrical \& Electronics Engineering from Dokuz Eylul University, Izmir, Turkey, and his $\mathrm{PhD}$ degree in Electrical and Computer Engineering from Clemson University, Clemson, SC, USA, in 1999 and 2007, respectively. Upon completion of his $\mathrm{PhD}$ degree, he worked as a post-doctoral research fellow in the Department of Electrical and Computer Engineering at Clemson University, then he joined the Department of Electrical \& Electronics Engineering at Izmir Institute of Technology, Izmir, Turkey, where he is currently an associate professor. His research interests include control and identification of time-delay systems, dynamic modelling of extensible continuum robot manipulators, nonlinear control techniques for kinematically redundant robot manipulators, partial state feedback and output feedback control, haptic systems and teleoperation, and learning, robust. and adaptive control of nonlinear systems.

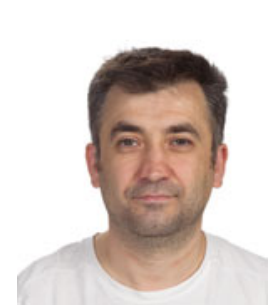

Erkan Zergeroglu received his BSc and MSc degrees in Electrical and Electronics Engineering from Hacettepe University (Ankara, Turkey) and Middle East Technical University (Ankara, Turkey) in 1992 and 1996, respectively. In 2000, he received his $\mathrm{PhD}$ degree in Electrical and Computer Engineering from Clemson University, Clemson, SC, USA. From 2000 to 2002, he worked for Lucent Technologies - Bell Labs Innovations, Optical Fiber Solutions, Sturbridge, MA, as a member of technical staff. In 2002, he joined the Computer Engineering Department of Gebze Institute of Technology, Kocaeli, Turkey, where he currently holds the position of associate professor. His main research interests are in the fields of model-based nonlinear control of electromechanical systems, design and application of partial state and output feedback control algorithms, and compensation of parametric uncertainties with robust, adaptive- and learning-type controllers.

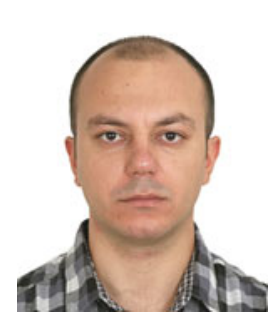

Alper Bayrak was born in Ankara, Turkey, on 19 October 1980 . He graduated from the Electrical and Electronics Engineering Department in Blacksea Technical University, Trabzon, Turkey, in 2004. He received his MSc degree in Electrical and Electronics Engineering from Gazi University and his $\mathrm{PhD}$ degree in Electronics and Communications Engineering from Izmir Institute of Technology, Izmir, Turkey. He worked as a research assistant at the Electrical and Electronics Department in Abant Izzet Baysal University, Bolu, Turkey, from 2005 to 2007, and at the Electrical and Electronics Department in Izmir Institute of Technology since from 2008 to 2014. Since 2014, he has been working as an assistant professor at the Electrical and Electronics Department in Abant Izzet Baysal University, Bolu, Turkey. His current fields of research are control, identification, nonlinear systems, and robotics.

\section{References}

Bidikli, B., Tatlicioglu, E., Bayrak, A., \& Zergeroglu, E. (2013). A new robust integral of sign of error feedback controller with adaptive compensation gain. In Proceedings of IEEE International Conference on Decision and Control (pp. 37823787), Florence: IEEE. 
Bidikli, B., Tatlicioglu, E., \& Zergeroglu, E. (2014). A self-tuning RISE controller formulation. In Proceedings of the American Control Conference (pp. 5608-5613), Portland, OR: IEEE.

Chen, J., Behal, A., \& Dawson, D.M. (2006). Adaptive output feedback control for a class of MIMO nonlinear systems. In Proceedings of American Control Conference (pp. 5300 5305), Minneapolis, MN: IEEE.

Chen, J., Behal, A., \& Dawson, D.M. (2008). Robust feedback control for a class of uncertain MIMO nonlinear systems. IEEE Transactions on Automatic Control, 53, 591-596.

Costa, R.R., Hsu, L., Imai, A.K., \& Kokotovic, P. (2003). Lyapunov-based adaptive control of MIMO systems. Automatica, 39, 1251-1257.

Gee, S.S., \& Wang, C. (2004). Adaptive neural control of uncertain MIMO nonlinear systems. IEEE Transactions on Neural Networks, 15, 674-692.

Khalil, H.K. (2002). Nonlinear systems (3rd ed. ). New York, NY: Prentice Hall.

Kosmatopoulos, E.B., \& Ioannou, P. (2002). Robust switching adaptive control of multi-input nonlinear systems. IEEE Transactions on Automatic Control, 47, 610-624.

Krstic, M., Kanellakopoulos, I., \& Kokotovic, P. (1995). Nonlinear and adaptive control design. New York, NY: John Wiley \& Sons.

Lee, D., Tatlicioglu, E., Burg, T.C., \& Dawson, D.M. (2008). Robust output tracking control of a surface vessel. In Proceedings of American Control Conference (pp. 544-549), Seattle, WA: IEEE.

Morse, A.S. (1993). A gain matrix decomposition and some of its applications. Systems \& Control Letters, 21, 1-10.

Reddy, K., Chen, J., Behal, A., \& Marzocca, P. (2007). Multiinput/multi-output adaptive output feedback control design for aeroelastic vibration suppression. AIAA Journal of Guidance, Control, and Dynamics, 30, 1040-1048.

Setlur, P., Wagner, J.R., Dawson, D.M., \& Chen, J. (2003). Nonlinear controller for automotive thermal management systems. In Proceedings of American Control Conference (pp. 2584 2589), Denver, CO: IEEE.

Slotine, J.J.E., \& Li, W. (1991). Applied nonlinear control. Englewood Cliffs, NJ: Prentice Hall.

Stepanyan, V., \& Kurdila, A. (2009). Asymptotic tracking of uncertain systems with continuous control using adaptive bounding. IEEE Transactions on Neural Networks, 20, 1320-1329.

Wang, Z., \& Behal, A. (2011). Continuous robust control for a class of uncertain MIMO nonlinear systems. In Proceedings of IEEE International Conference on Decision and Control and European Control Conference (CDCECC) (pp. 75617566), Orlando, FL: IEEE.

Wang, Z., Behal, A., Xian, B., \& Chen, J. (2011). Lyapunov-based adaptive control design for a class of uncertain MIMO nonlinear systems. In Proceedings of International Symposium on Intelligent Control Part of IEEE Multi-Conference on Systems and Control (pp. 1510-1515), Denver, CO: IEEE.

Wang, Z., Chen, J., \& Behal, A. (2010). Robust adaptive control design for a class of MIMO nonlinear systems. In Proceedings of International Symposium on Intelligent Control Part of IEEE Multi-Conference on Systems and Control (pp. 22842289), Yokohama: IEEE.

Xian, B., Dawson, D.M., de Queiroz, M.S., \& Chen, J. (2004). A continuous asymptotic tracking control strategy for uncertain nonlinear systems. IEEE Transactions on Automatic Control, 49, 1206-1211.

$\mathrm{Xu}$, H., \& Ioannou, P.A. (2003). Robust adaptive control for A class of MIMO nonlinear systems with guaranteed error bounds. IEEE Transactions on Automatic Control, 48, 728742 .
Zergeroglu, E., Dawson, D.M., de Queiroz, M.S., \& Behal, A. (2001). Vision-based nonlinear tracking controllers with uncertain robot-camera parameters. IEEE/ASME Transactions on Mechatronics, 6, 322-337.

Zhang, X.T., Dawson, D.M., de Queiroz, M.S., \& Xian, B. (2004). Adaptive control for a class of MIMO nonlinear systems with non-symmetric input matrix. In Proceedings of American Control Conference (pp. 1324-1329), Taipei: IEEE.

\section{Appendix 1. Proof of Lemma 4.2}

Proof: First, we note that if $e_{n, i}(t) \equiv 0$ on some interval, then $\dot{e}_{n, i}(t) \equiv 0$ on the same interval, and the inequality (60) yields this qualification. Therefore, without loss of generality, we assume that $e_{n, i}(t)$ is absolutely greater than zero on the interval of $\left[t_{0}, t\right]$. Let $T \in\left[t_{0}, t\right)$ be the last instant of time when $\dot{e}_{n, i}(t)$ changes sign. Then, on the interval $[T, t], \dot{e}_{n, i}(t)$ has a constant sign, hence

$$
\int_{T}^{t}\left|\dot{e}_{n, i}(\sigma)\right| d \sigma=\left|\int_{T}^{t} \dot{e}_{n, i}(\sigma) d \sigma\right|=\left|e_{n, i}(t)-e_{n, i}(T)\right|
$$

From the boundedness of the function $\dot{e}_{n, i}(t)$, it follows that there exists a constant $\gamma>0$ such that $\left|\dot{e}_{n, i}(t)\right| \leq \gamma$; therefore,

$$
\int_{t_{0}}^{T}\left|\dot{e}_{n, i}(\sigma)\right| d \sigma \leq \gamma\left(T-t_{0}\right)
$$

On the other hand, we obtain the following equality from the application of the mean value theorem in (Khalil, 2002):

$$
\int_{t_{0}}^{T}\left|e_{n, i}(\sigma)\right| d \sigma=e_{n, i_{*}}\left(T-t_{0}\right)
$$

where $e_{n, i_{*}}$ is some constant intermediate value of $\left|e_{n, i}(t)\right|$ on the interval $\left[t_{0}, T\right]$. By assumption, $e_{n, i_{*}}$ is bounded away from zero. Therefore, by using inequality (A2) and equality (A3), we can conclude as follows:

$$
\int_{t_{0}}^{T}\left|\dot{e}_{n, i}(\sigma)\right| d \sigma \leq \gamma_{2} \int_{t_{0}}^{T}\left|e_{n, i}(\sigma)\right| d \sigma
$$

where $\gamma_{2}=\gamma / e_{n, i_{*}}$. Combining the relationships in (A1) and (A4), we can write

$$
\int_{t_{0}}^{t}\left|\dot{e}_{n, i}(\sigma)\right| d \sigma \leq\left|e_{n, i}(t)\right|+\left|e_{n, i}(T)\right|+\gamma_{2} \int_{t_{0}}^{T}\left|e_{n, i}(\sigma)\right| d \sigma
$$

which yields the inequality (60) with definition $\gamma_{1} \triangleq$ $\sup \left|e_{n, i}(T)\right|$.

\section{Appendix 2. Proof of Lemma 4.3}

Proof: We start our analysis by integrating (61) in time from $t_{0}$ to $t$

$$
\begin{aligned}
\int_{t_{0}}^{t} L(\sigma) d \sigma= & \int_{t_{0}}^{t} e_{n}^{T}(\sigma) \alpha^{T}\left(\bar{N}(\sigma)-C \operatorname{Sgn}\left(e_{n}(\sigma)\right)\right) d \sigma \\
& -\int_{t_{0}}^{t} e_{n}^{T}(\sigma) \alpha^{T} \Omega(\sigma) C \operatorname{Sgn}\left(e_{n}(\sigma)\right) d \sigma
\end{aligned}
$$




$$
\begin{aligned}
& +\int_{t_{0}}^{t} \dot{e}_{n}^{T}(\sigma) \bar{N}(\sigma) d \sigma \\
& -\int_{t_{0}}^{t} \dot{e}_{n}^{T}(\sigma) \Omega(\sigma) C \operatorname{Sgn}\left(e_{n}(\sigma)\right) d \sigma \\
& -\int_{t_{0}}^{t} \dot{e}_{n}^{T}(\sigma) C \operatorname{Sgn}\left(e_{n}(\sigma)\right) d \sigma
\end{aligned}
$$

where (16) was utilised. To ease the presentation, we will consider each term on the right-hand side of (B1) separately. The first term:

$$
\begin{aligned}
& \int_{t_{0}}^{t} e_{n}^{T}(\sigma) \alpha^{T}\left(\bar{N}(\sigma)-C \operatorname{Sgn}\left(e_{n}(\sigma)\right)\right) d \sigma \\
& \quad=\int_{t_{0}}^{t} \sum_{i=1}^{m} \alpha_{i} e_{n, i}(\sigma)\left(\bar{N}_{i}(\sigma)-C_{i} \operatorname{sgn}\left(e_{n, i}(\sigma)\right)\right) d \sigma \\
& \quad \leq \sum_{i=1}^{m} \alpha_{i}\left(\zeta_{\bar{N}_{i}}-C_{i}\right) \int_{t_{0}}^{t}\left|e_{n, i}(\sigma)\right| d \sigma .
\end{aligned}
$$

The second term:

$$
\begin{aligned}
& -\int_{t_{0}}^{t} e_{n}^{T}(\sigma) \alpha^{T} \Omega(\sigma) C \operatorname{Sgn}\left(e_{n}(\sigma)\right) d \sigma \\
& =-\int_{t_{0}}^{t} \sum_{i=1}^{m-1} \alpha_{i} e_{n, i}(\sigma) \sum_{j=i+1}^{m} C_{j} \Omega_{i, j}(\sigma) \operatorname{sgn}\left(e_{n, j}(\sigma)\right) d \sigma \\
& \leq \sum_{i=1}^{m-1} \sum_{j=i+1}^{m} \alpha_{i} C_{j} \zeta_{\Omega_{i, j}} \int_{t_{0}}^{t}\left|e_{n, i}(\sigma)\right| d \sigma
\end{aligned}
$$

The third term:

$$
\begin{aligned}
& \int_{t_{0}}^{t} \dot{e}_{n}^{T}(\sigma) \bar{N}(\sigma) d \sigma \\
& \quad=\int_{t_{0}}^{t} \sum_{i=1}^{m} \dot{e}_{n, i}^{T}(\sigma) \bar{N}_{i}(\sigma) d \sigma \\
& \quad \leq \sum_{i=1}^{m} \zeta_{\bar{N}_{i}} \int_{t_{0}}^{t}\left|\dot{e}_{n, i}(\sigma)\right| d \sigma \\
& \quad \leq \sum_{i=1}^{m} \zeta_{\bar{N}_{i}}\left(\gamma_{1}+\gamma_{2} \int_{t_{0}}^{t}\left|e_{n, i}(\sigma)\right| d \sigma+\left|e_{n, i}\right|\right) .
\end{aligned}
$$

The fourth term:

$$
\begin{aligned}
& -\int_{t_{0}}^{t} \dot{e}_{n}^{T}(\sigma) \Omega(\sigma) C \operatorname{Sgn}\left(e_{n}(\sigma)\right) d \sigma \\
& =-\int_{t_{0}}^{t} \sum_{i=1}^{m-1} \dot{e}_{n, i}(\sigma) \sum_{j=i+1}^{m} C_{j} \Omega_{i, j}(\sigma) \operatorname{sgn}\left(e_{n, j}(\sigma)\right) d \sigma
\end{aligned}
$$

$$
\begin{aligned}
& \leq \sum_{i=1}^{m-1} \sum_{j=i+1}^{m} C_{j} \zeta_{\Omega_{i, j}} \int_{t_{0}}^{t}\left|\dot{e}_{n, i}(\sigma)\right| d \sigma \\
& \leq \sum_{i=1}^{m-1} \sum_{j=i+1}^{m} C_{j} \zeta_{\Omega_{i, j}}\left(\gamma_{1}+\gamma_{2} \int_{t_{0}}^{t}\left|e_{n, i}(\sigma)\right| d \sigma+\left|e_{n, i}\right|\right) .
\end{aligned}
$$

The fifth term:

$$
\begin{aligned}
& -\int_{t_{0}}^{t} \dot{e}_{n}^{T}(\sigma) C \operatorname{Sgn}\left(e_{n}(\sigma)\right) d \sigma \\
& =-\int_{t_{0}}^{t} \sum_{i=1}^{m} C_{i} \dot{e}_{n, i}(\sigma) \operatorname{sgn}\left(e_{n, i}(\sigma)\right) d \sigma \\
& =-\sum_{i=1}^{m} C_{i} \int_{t_{0}}^{t} \operatorname{sgn}\left(e_{n, i}(\sigma)\right) d\left(e_{n, i}\right) \\
& =-\sum_{i=1}^{m} C_{i} \int_{t_{0}}^{t} d\left(\left|e_{n, i}\right|\right) \\
& =-\sum_{i=1}^{m} C_{i}\left|e_{n, i}(t)\right|+\sum_{i=1}^{m} C_{i}\left|e_{n, i}\left(t_{0}\right)\right| .
\end{aligned}
$$

It is noted that, the result of Lemma 4.2 was utilised to obtain the last lines of (B4) and (B5). After combining the upper bounds in (B2)-(B6), we obtain

$$
\begin{aligned}
\int_{t_{0}}^{t} L & (\sigma) d \sigma \\
\leq & \sum_{i=1}^{m-1} \alpha_{i}\left[\left(1+\frac{\gamma_{2}}{\alpha_{i}}\right)\left(\zeta_{\bar{N}_{i}}+\sum_{j=i+1}^{m} \zeta_{\Omega_{i, j}} C_{j}\right)-C_{i}\right] \\
& \times \int_{t_{0}}^{t}\left|e_{n, i}(\sigma)\right| d \sigma \\
& +\alpha_{m}\left[\left(1+\frac{\gamma_{2}}{\alpha_{m}}\right) \zeta_{\bar{N}_{m}}-C_{m}\right] \int_{t_{0}}^{t}\left|e_{n, m}(\sigma)\right| d \sigma \\
& +\left(\zeta_{\bar{N}_{m}}-C_{m}\right)\left|e_{n, m}\right| \\
& +\sum_{i=1}^{m-1}\left(\zeta_{\bar{N}_{i}}+\sum_{j=i+1}^{m} \zeta_{\Omega_{i, j}} C_{j}-C_{i}\right)\left|e_{n, i}\right| \\
& +\gamma_{1} \sum_{i=1}^{m-1} \sum_{j=i+1}^{m} \zeta_{\Omega_{i, j}} C_{j}+\gamma_{1} \sum_{i=1}^{m} \zeta_{\bar{N}_{i}}+\sum_{i=1}^{m} C_{i}\left|e_{n, i}\left(t_{0}\right)\right|
\end{aligned}
$$

Based on (B7), we first choose $C_{m}$ to satisfy (62) to make second and third expressions on the right-hand side negative, we next choose $C_{i}$ starting from $(m-1)$ with a decreasing order to satisfy (62) to make the first and fourth expressions on the right-hand side negative, and finally, we utilised the definition of $\zeta_{L}$ in (64) to obtain (63), thus completing the proof of Lemma 4.3. 